\title{
Eficiencia fisiológica de uso de NPK en clones autoincompatible y autocompatible de cacao ( Theobroma cacao L.) en Colombia
}

\section{Physiological efficiency of use NPK self- compatible and self-incompatible cacao clone (Theobroma cacao L.) in Colombia}

\author{
Eficiência fisiológica do uso de NPK em clones \\ não auto compatíveis e auto compatíveis de cacau \\ (Theobroma cacao L.) na Colômbia
}

\begin{abstract}
${ }^{1}$ Yina Jazbleidi Puentes-Páramo, ${ }^{2}$ Juan Carlos Menjivar-Flores \& ${ }^{3}$ Antonio María Ortíz Camacho
IIngeniera Agrónoma, Magister en Biotecnología de plantas, Doctora en Ciencias Agropecuarias. 'Ingeniero Agrónomo, Magister en Suelos y Aguas, Doctor en Ciencia del Suelo. ${ }^{3}$ Ingeniero Agrónomo

1,2 Facultad de Ciencias Agropecuarias. Universidad Nacional de Colombia Sede Palmira. Valle, Colombia. ${ }^{3}$ Fondo Nacional del Cacao. Producción e Investigación. FEDECACAO, Cali. Valle, Colombia.
\end{abstract}

1yjpuentesp@unal.edu.co, 2jcmenjivarf@unal.edu.co, 3antonioortizc@fedecacao.co

\section{Resumen}

La investigación tuvo como objetivo evaluar la eficiencia fisiológica de N-P-K en clones de cacao de sabor y aroma autocompatible (CCN-51, ICS-95) y autoincompatible: (TSH-565, ICS-39), con la finalidad de determinar la capacidad de producción de grano seco por unidad de nutriente absorbido. Se utilizó un diseño en bloques completos al azar con cinco tratamientos y cuatro repeticiones, los tratamientos fueron diseñados a partir del nivel de fertilidad natural del suelo (TR-Tratamiento de referencia) y sobre este el 25, 50, 75 y 100\% más de Nitrógeno-Fósforo-Potasio (N-P-K), correspondiente al T1, T2, T3 y T4. Los resultados evidencian que hay diferencias altamente significativas entre clones para los nutrientes N-P-K absorbidos, exhibiendo mayor absorción de nitrógeno, seguido de potasio y por ultimo fósforo, en este sentido, el clon autocompatible CCN-51 mostro mayor habilidad de absorción para la mayoría de nutrientes, excepto fósforo, sin embargo, no es generalizada la mayor habilidad para clones autocompatibles con respecto a clones autoincompatibles. La mayor eficiencia fisiológica de uso de nutrientes N-P-K se presentó en el tratamiento uno y la menor capacidad de producción de grano seco de cacao por unidad de nutriente se presentó con la dosis nutricional más alta en el tratamiento cuatro, lo cual sugiere que a mayor dosis disminuye la eficiencia fisiológica de uso de nitrógeno, fósforo y potasio de los clones.

Palabras clave: fertilización, eficiencia de uso de nutrientes, nutrición mineral 


\section{Abstract}

The research aimed to evaluate the physiological efficiency of N-P-K in clones of cocoa flavor and self-compatible flavor (CCN-51, ICS-95) and selfincompatible (TSH-565, ICS-39), to determine the ability of dry grain production per unit of absorbed nutrient. The design was randomized complete blocks with five treatments and four replications, the treatments were designed from the level of natural soil fertility (TR-treatment baseline) and this 25, 50, 75 and 100\% more nitrogen-PhosphorusPotassium (N-P-K), corresponding to T1, T2, T3 and T4. The results show that there are highly significant differences between clones for N-P-K nutrients absorbed, exhibiting increased uptake of nitrogen, followed potassium and last match, in this sense, the self-compatible clone $\mathrm{CCN}-51$ showed greater ability to absorb for most nutrients, except phosphorus, however, it is not generally the greatest ability to self-compatible clones with respect to self-incompatible clones. Most physiological efficiency of nutrient use N-P-K appeared in treating one and the lowest production capacity of dry cocoa beans per nutrient unit was presented with the highest nutritional doses in the treatment four, which suggests that higher doses decreases the physiological efficiency of use of nitrogen, phosphorus and potassium clones.

Key-words: fertilization, mineral nutrition, nutrient use efficiency.

\section{Introducción}

El cacao (Theobroma cacao L.) es un cultivo con mucho futuro en países como Costa de Marfil, Ghana, Colombia, Ecuador y Brasil, hoy en día los precios en los mercados internacionales son muy atractivos si de sabor y aroma se trata por supuesto, en ese sentido nuevas áreas se dedican a la producción, con el inconveniente de no contar con estudios que demuestren la eficiencia de los diferentes variedades y clones en el uso de los nutrientes, es así como Akinrinde (2006) afirma que la prioridad de los productores de las zonas tropicales y subtropicales

\section{Resumo}

A pesquisa teve como objetivo avaliar a eficiência fisiológica de N-P-K em clones de cacau, de sabor e aroma auto compatível (CCN-51, ICS-95) e não auto compatível: (TSH-565, ICS-39), com a finalidade de determinar a capacidade de produção de grão seco por unidade de nutriente absorvido. Utilizou-se um delineamento de blocos ao acaso com cinco tratamentos e quatro repetições. Os tratamentos foram escolhidos baseados no nível de fertilidade natural do solo (TR-Tratamento de referência) e sobre ele 25, 50, 75 e 100\% mais de Nitrogênio-Fósforo-Potássio (N-P-K), correspondendo a T1, T2, T3 e T4. Os resultados mostram que existem diferenças altamente significativas entre clones para os nutrientes N-P-K absorvidos, exibindo maior absorção de nitrogênio, depois potássio e finalmente fósforo, assim, o clone auto compatível CCN-51 mostrou maior habilidade de absorção para a maioria dos nutrientes, exceto o fósforo. No entanto, não pode ser generalizada a maior habilidade dos clones auto compatíveis quando comparados com clones não auto compatíveis. A maior eficiência fisiológica do uso de nutrientes N-P-K foi observada no tratamento 1 e a menor capacidade de produção de grão seco de cacau por unidade de nutriente foi observada no tratamento quatro, sugerindo isso que quanto maior a dose, menor a eficiência fisiológica do uso do nitrogênio, fósforo e potássio dos clones.

Palavras-chave: Fertilização, Eficiência do uso de nutrientes, nutrição mineral es garantizar la sostenibilidad de la producción, aunque para ello se tenga que hacer un uso excesivo de fertilizantes; en el mismo sentido Lija et al. (2014) afirman que los fertilizantes minerales son una fuente alternativa para suplir nutrientes al suelo, mejorando la productividad de los cultivos, sin embargo una inadecuada aplicación se traduce en un consumo.

Lograr incrementos en la producción por efecto de la adición de fertilizantes es una práctica común a 
muchos cultivos, sin embargo utilizar plantas eficientes en el uso de los nutrientes es una estrategia muy importante para reducir los costos de producción relacionados con el empleo de estos productos (Kumar, 2000). La evaluación del uso eficiente de nutrientes nos lleva a identificar genotipos y cultivares por su habilidad en la absorción y utilización de los nutrientes que se traducen en buenos rendimientos en campo (Baligar, 2001); es así como la evaluación del uso eficiente que los cultivos hacen de los nutrientes cobra importancia, sin embargo, los estudios se enfocan mucho a la dinámica de la aplicación de N-P-K y su efecto en el rendimiento (Puentes et al., 2014a).

Algunos autores se han dedicado al estudio del uso eficiente de nutrientes en cultivos de ciclo corto como sen maíz, arroz, trigo, papa y otros (Xu et al., 2015; Soratto et al., 2015), los resultados obtenidos en corto tiempo pueden ser adoptados por los productores, sin embargo, en cacao, un cultivo perenne, el tiempo limita obtener información rápidamente. Ribeiro et al. (2008) evaluaron el uso eficiente de nutrientes en condiciones de invernadero y plantas de cacao de siete meses de edad, los resultados limitan su aplicación a cultivos en producción; por otra parte, Puentes et al. (2014a) evaluaron el uso eficiente de nutrientes en función de la eficiencia agronómica y de recuperación de $\mathrm{N}-\mathrm{P}-\mathrm{K}$ en condiciones de campo y con plantaciones en producción, más no se estudió la eficiencia fisiológica de los mismos.

La eficiencia fisiológica se refiere al aumento en $\mathrm{kg}$ del rendimiento por kg de nutriente absorbido (Roberts, 2008); muchos factores influyen en la misma, sobre todo los relacionados con el suelo, tales como: el tipo de fertilizantes, clima, manejo agronómico y cultivo, especialmente lo concerniente con el desarrollo del sistema radical, ya que se relaciona con la toma de nutrientes y el uso de los mismos por las plantas (Fan et al., 2010).

El cacao (T. cacao L.) se cultiva en suelos muy diversos, y en Colombia existen aproximadamente 157.000 hectáreas sembradas con diferentes clones y variedades, en los cuales la aplicación de fertilizantes se basa en recomendaciones obtenidas a partir de los análisis de suelos y de ensayos de fertilidad (Mora et al., 2011), sin embargo, no existen datos relacionados con la eficiencia fisiológica en el uso de N-P-K, lo cual es un gran vacío en el conocimiento, ya que clones de cacao eficientes en el uso de nutrientes, representan al agricultor mayores ingresos y una contribución al uso sostenible de los recursos naturales. En ese sentido es importante el aporte de esta investigación que tuvo como objetivo determinar la eficiencia fisiológica de uso de N-P-K en clones de cacao de sabor y aroma autocompatibles y autoincompatibles, CCN-51, ICS-95, TSH565 y ICS-39 respectivamente, con fines de caracterizar la habilidad en producir grano por unidad de nutriente absorbido por cada clon.

\section{Materiales y métodos}

La investigación se realizó en el Centro Experimental de la Federación Nacional de Cacaoteros a 1120 msnm, ubicado geográficamente en el municipio de Miranda del departamento del Cauca (Colombia) a $3^{\circ} 23^{\prime} 44^{\prime \prime}$ Latitud Norte y 76³0'33" de Latitud Oeste. Los clones de cacao (T. cacao L.) de sabor y aroma establecidos en campo con cinco años de edad y evaluados en la presente investigación fueron: ICS-39, TSH-565, ICS-95 y CCN-51, dos clones autoincompatibles y dos autocompatibles respectivamente (Puentes et al., 2014b).

Al inicio de la investigación se determinaron las características físicas y químicas del suelo según metodologías estandarizadas por el laboratorio del Centro Internacional de Agricultura Tropical (CIAT) e interpretadas con la quinta aproximación (ICA, 1992). De acuerdo en estos análisis se diseñaron los tratamientos con base al nivel de fertilidad natural del suelo (TR). El TR se determinó a partir de la concentración de N-P-K del suelo en kg.ha-1 (4923,5-146,5); el resto de los tratamientos fueron incrementos del $25 \%, 50 \%, 75 \%$ y $100 \%$ de $\mathrm{N}-\mathrm{P}_{2} \mathrm{O}_{5 y}$ $\mathrm{K}_{2} \mathrm{O}$ sobre el nivel de fertilidad natural del suelo (TR) dando lugar al T1 (61-29,3-183), T2 (73-35,2-219,7), T3 $(86-41-256,4)$ y T4 $(98-47-293)$ respectivamente; en los cuales se utilizó las fuentes de fertilización: 
urea $\mathrm{CO}\left(\mathrm{NH}_{2}\right)_{2}$ (46-00-00), Fosfato diamónico $\left(\mathrm{NH}_{4}\right)_{2}\left(\mathrm{HPO}_{4}\right)$ (18-46-00) y nitrato de potasio $\mathrm{KNO}_{3}$ (13-00-44). Los tratamientos se aplicaron en forma fraccionada, así, el $50 \%$ en mayo y el otro $50 \%$ en septiembre, en los años 2010, 2011 y 2012.

El rendimiento se estimó mediante conteo y pesado de todos los frutos (mazorca) obtenidos en cada cosecha por clon por año, por consiguiente, los granos se extrajeron en forma manual, luego se contaron y pesaron en húmedo, a partir de esta información se determinó el índice de grano (IG) en gramos a través de la fórmula propuesta por Allen (1987), donde el IG es igual al producto del peso total de granos/mazorca por el factor 0,38 y dividido entre el número de granos/mazorca (Puentes et al., 2014b). En este sentido, el rendimiento es el producto del índice de grano, del número de mazorcas por árbol, el número de grano por mazorca y la densidad de siembra.

Para determinar las concentraciones de NPK en almendras, se muestrearon siete mazorcas maduras por cada árbol de cada tratamiento, y con los granos se conformó una muestra representativa, las cuales fueron procesadas en el CIAT siguiendo protocolos estándar, así, $\mathrm{N}$ se determinó por el método Kjheldhal; $\mathrm{P}$ y K $\mathrm{K}^{+}$por descomposición vía seca (550 ${ }^{\circ} \mathrm{C}$ ) y mineralización con $\mathrm{HCl} 6 \mathrm{M}$, cuantificados por colorimetría y absorción atómica respectivamente.

La eficiencia fisiológica (EF) se define como el incremento del rendimiento obtenido en kilogramo por cada kilogramo de nutriente absorbido. Así, la EF de uso para NPK se determinó según la ecuación (1) utilizada por Fageria et al. (2010).

$k g-f)=R(f)-R(t) C N(f)-C N(t)(1)$

En donde, $R(f)$ : rendimiento del grano del tratamiento evaluado; $R(t)$ : rendimiento de grano del TR; $C N(f)$ : cantidad del nutriente en la almendra del tratamiento evaluado, y $C N(t)$ : cantidad del nutriente en la almendra del tratamiento testigo.

El diseño experimental consistió en bloques completos al azar con cinco tratamientos y cuatro repeticiones. La unidad experimental consistió en cuatro árboles de cacao, sembrados a $3 \mathrm{~m} \times 3 \mathrm{~m}$ entre plantas $\mathrm{x} 4 \mathrm{~m}$ entre calle, para una densidad de 952 plantas por ha, la unidad de muestreo para determinar concentración de nutrientes fue de 7 mazorcas de cacao totalmente maduras por repetición de cada tratamiento. Los datos fueron analizados con SPSS 20 (IBM, 2011), y consistió en análisis de varianza (Andeva) para determinar diferencias entre las variables de respuesta; posteriormente se realizaron pruebas de comparación de medias (Tukey).

\section{Resultados y discusión}

\section{Análisis del suelo experimental}

Los datos obtenidos en el análisis químico y físico del suelo experimental fueron: $\mathrm{pH}$ de 6,4 (acidez débil), alta materia orgánica con $7,4 \%$, contenido medio de potasio $(0,26 \mathrm{cmol} / \mathrm{kg})$, alto para calcio $(9,73 \mathrm{cmol} / \mathrm{kg})$ y magnesio $(7,9 \mathrm{cmol}, \mathrm{kg})$, fósforo $(8,6$ ppm) y el resto de los elementos químicos (datos no publicados) fueron normales, así mismo, porosidad superior a $55 \%$, textura franco limoso con presencia de arcillas illitas, con interestratificados illita/ vermiculita en grandes proporciones, y densidad aparente de 1,2 Mg/m3, adecuadas para el cultivo de cacao (Puentes et al., 2014b); los cuales se encuentran dentro de los rangos reportados como normales para cacao por García (2007), presentando condiciones homogéneas y adecuadas para el desarrollo del cultivo.

\section{Rendimiento}

El rendimiento mostro diferencias altamente significativas $(p<0.001)$ por efecto de los tratamientos y tipo de clon (Puentes et al.,2016); así, el clon CCN51 obtuvo el mayor rendimiento en el tratamiento T1 (2020 kg ha-1), seguido el clon ICS-39 (1634 kg ha-1 $^{-1}$, TSH-565(1340 kg ha-1) e ICS-95(1337 kg ha-1) en el tratamiento T2; así, los rendimientos obtenidos muestran un diferencial del $34 \%$ para los clones ICS-95 y TSH-565 y del 19\% para el clon ICS-39 con respecto al clon de mayor rendimiento $(\mathrm{CCN}$ 51), exhibiendo en forma generalizada un menor rendimiento para los clones auto-incompatibles como los sugiere Mora et al. (2011). 
Los menores rendimientos se presentaron en el tratamiento TR para todos los clones evaluados, comportamiento normal debido a la ausencia de fertilización, como lo corrobora Aguilar et al. (2015); lo que sugiere que el contenido nutricional del suelo (TR) no satisface las necesidades nutricionales del cultivo y por lo tanto es necesario fertilizar. Sin embargo, después del tratamiento T2 es notorio que se deprime el rendimiento, similares resultados se presentaron para otros cultivos tales como en uva (Puerto et al., 2014) y caña de azúcar (Rasche et al., 2012).
La relación entre rendimiento y nutrientes absorbidos por la planta (en almendra) para el tratamiento con mayor rendimiento por cada clon (Figura 1), muestra que a mayor rendimiento existe mayor absorción de nutrientes en almendra, y en mayor proporción para nitrógeno, seguido de potasio y por ultimo fósforo (Puentes et al., 2014b). El clon autocompatible CCN51, con el mayor rendimiento, mostro mayor habilidad en absorción de N y P, excepto K; el clon autoincompatible ICS-39 mostro mayor habilidad en absorción de K. Sin embargo, no evidencia una clara superioridad en absorción de nutrientes N-P-K para autocompatibles con respecto a clones autoincompatibles.

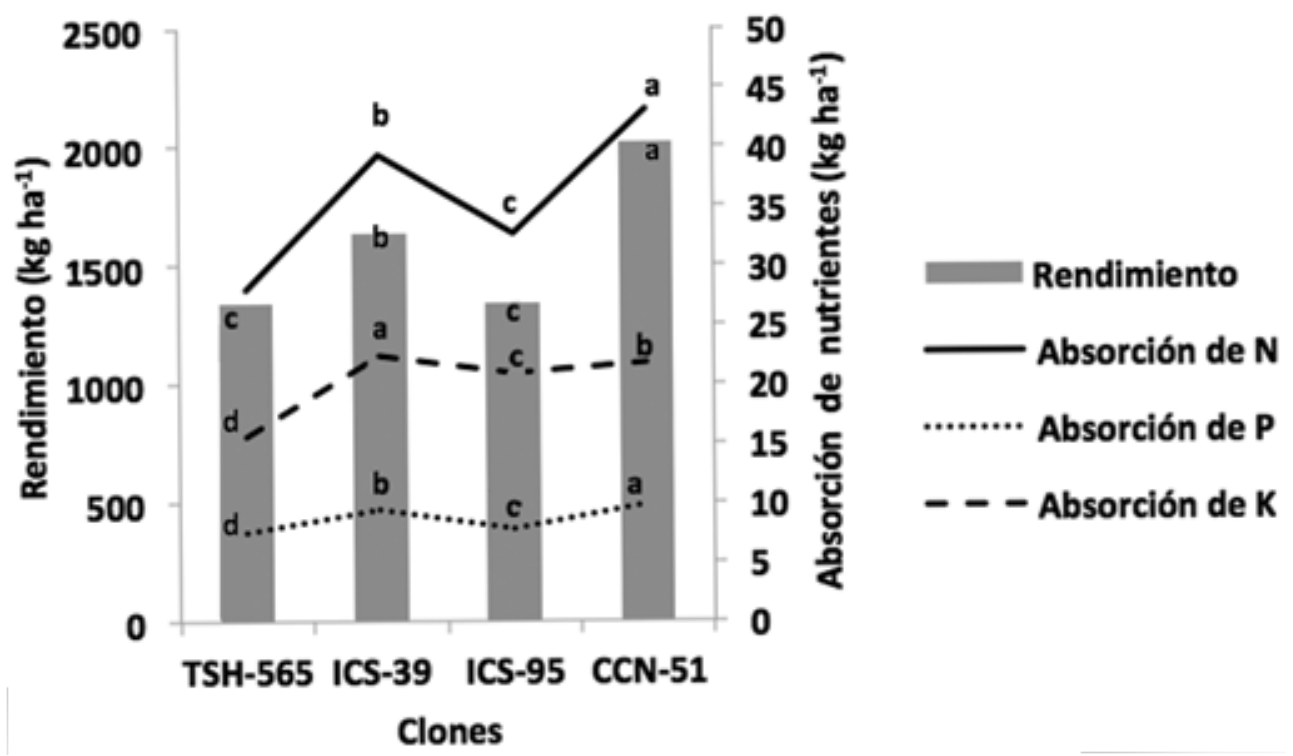

Figura 1. Relación entre rendimiento y nutrientes absorbidos por la planta para cada clon Valores con letra distinta difieren estadísticamente según la prueba de Tukey $(p \leq 0,05)$

Así mismo, se evidencia la habilidad de cada clon en la absorción de nutrientes y su capacidad para aumentar el rendimiento, como se presenta en los clones TSH-565 e ICS-95 con rendimientos similares y con diferente absorción de $\mathrm{N}$, en este sentido, el clon ICS-95 (32,66 kg ha-1) con mayor absorción de $\mathrm{N}$ que el clon TSH-565 (27,95 kg ha-1), no refleja esa absorción adicional (15\%) en el rendimiento, lo que sugiere un potencial de absorción diferencial para cada clon.

\section{Eficiencia fisiológica de uso de NPK en clones de cacao}

La eficiencia fisiológica de uso de nitrógeno, fósforo y potasio mostraron diferencias altamente significativas $(p<0.001)$ entre clones, similares resultados obtuvo Kumar (2000). Las figuras 2, 3 y 4 muestran la eficiencia fisiológica de uso de Nitrógeno, fósforo y potasio respectivamente, de los cuatro clones evaluados bajo diferentes dosis de fertilización ( $T 1$, T2, T3 y T4), con sus respectivas ecuaciones y coeficientes de determinación $\left(\mathrm{R}^{2}\right)$. 
La mayor eficiencia fisiológica de uso del nitrógeno (EFN) se presentó para el clon $\mathrm{CCN}-51$ en el tratamiento $\mathrm{T} 1\left(45,3 \mathrm{~kg} \mathrm{~kg}^{-1}\right)$, lo cual significa que por cada kilogramo de nitrógeno absorbido se producen $45,3 \mathrm{~kg}$ de grano seco de cacao.
Posteriormente, el clon ICS-39 en el tratamiento T3 $\left(40,87 \mathrm{~kg} \mathrm{~kg}^{-1}\right)$, el clon TSH-565 en el T2 $(40,86$ $\left.\mathrm{kg} \mathrm{kg}^{-1}\right)$ y por último el clon ICS-95 en el T3 $(40,38$ $\left.\mathrm{kg} \mathrm{kg}^{-1}\right)$. La menor EFN se presentó en el T4 para todos los clones.

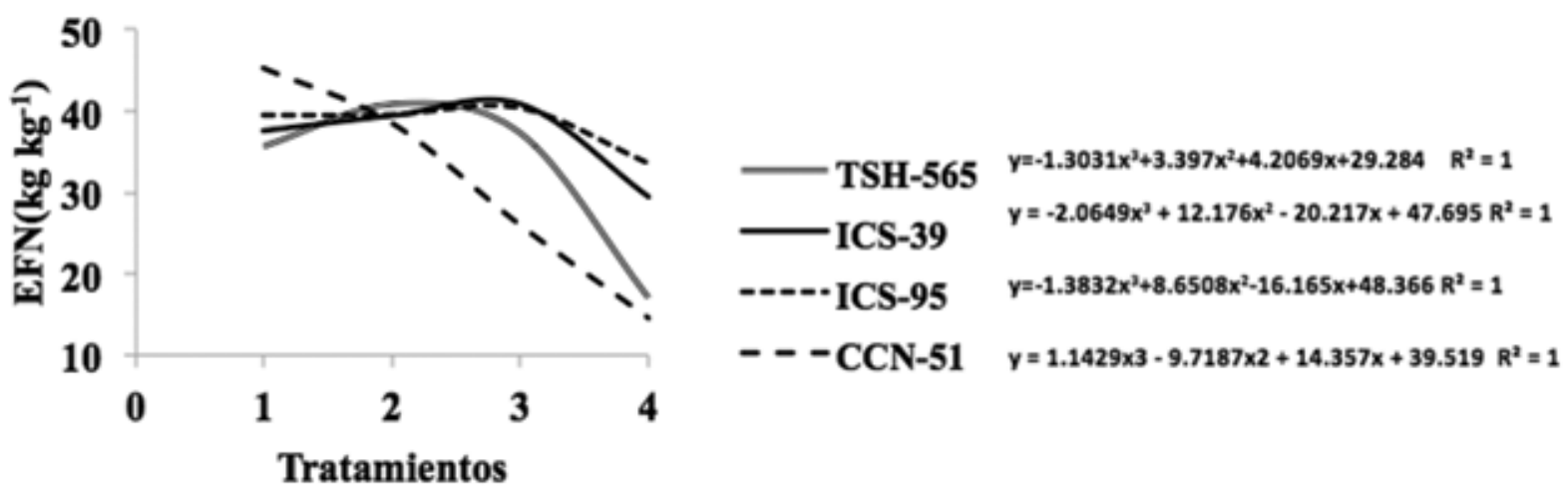

Figura 2. Eficiencia fisiológica de uso del nitrógeno (EFN) para los clones TSH-565, ICS-39, ICS-95 y CCN-51

El clon CCN-51 mostró la mayor EFN, como también, la menor EFN (14,59 $\left.\mathrm{kg} \mathrm{kg}^{-1}\right)$ en el T4 con respecto a los demás clones. Lo que sugiere que las altas dosis de nitrógeno (T4) disminuyen la eficiencia fisiológica de uso del nutriente, como lo sugiere Rouanet (1994).

La mayor eficiencia fisiológica de uso del fósforo (EFP) se presentó para el clon ICS-39 en el tratamiento T1 $\left(195,45 \mathrm{~kg} \mathrm{~kg}^{-1}\right)$, lo cual significa que por cada kilogramo de fósforo absorbido se producen $195,45 \mathrm{~kg}$ de grano seco de cacao. Posteriormente, el clon TSH-565 en el tratamiento T3 $\left(177,11 \mathrm{~kg} \mathrm{~kg}^{-1}\right)$, el clon ICS-95 en el T2 $(173,9 \mathrm{~kg}$ $\mathrm{kg}^{-1}$ ) y por último el clon CCN-51 en el T2 $(173,35$ $\left.\mathrm{kg} \mathrm{kg}^{-1}\right)$. La menor EFP se presentó en el T4 para todos los clones.

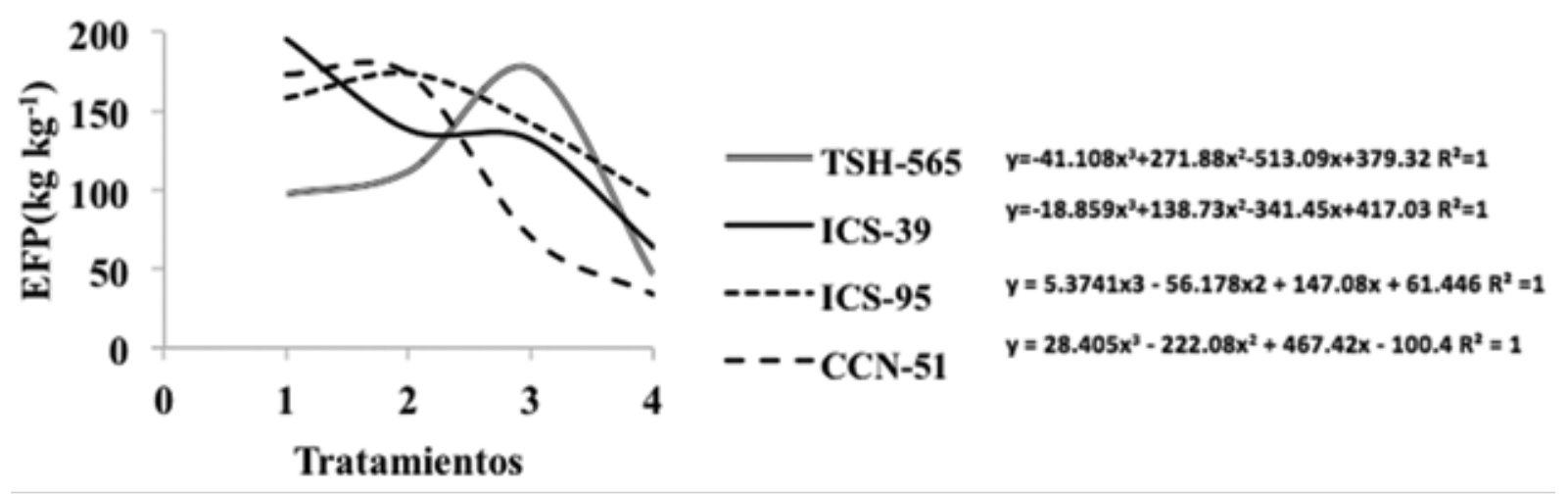

Figura 3. Eficiencia fisiológica de uso del fósforo (EFP) para los clones TSH-565, ICS-39, ICS-95 y CCN-51 
El clon ICS-39 mostro la mayor EFP, sin embargo, la menor EFP la presento el clon CCN-51 $(33,90 \mathrm{~kg}$ $\mathrm{kg}^{-1}$ ) en el T4 con respecto a los demás clones. En tanto, es claro que las altas dosis de fósforo (T4) para los clones evaluados disminuyen la eficiencia fisiológica de uso del nutriente.

La mayor eficiencia fisiológica de uso del potasio $(E F K)$ se presentó para el clon $\mathrm{CCN}-51$ en el tratamiento $\mathrm{T} 1\left(76,57 \mathrm{~kg} \mathrm{~kg}^{-1}\right)$, lo cual significa que por cada kilogramo de nitrógeno absorbido se producen $76,57 \mathrm{~kg}$ de grano seco de cacao. Posteriormente, el clon ICS-39 en el tratamiento T3 $\left(74,05 \mathrm{~kg} \mathrm{~kg}^{-1}\right)$, el clon TSH-565 en el T3 $(70,29$ $\left.\mathrm{kg} \mathrm{kg}^{-1}\right)$ y por último el clon ICS-95 en el T2 $(55,64$ $\left.\mathrm{kg} \mathrm{kg}^{-1}\right)$. La menor EFK se presentó en el T4 para todos los clones.

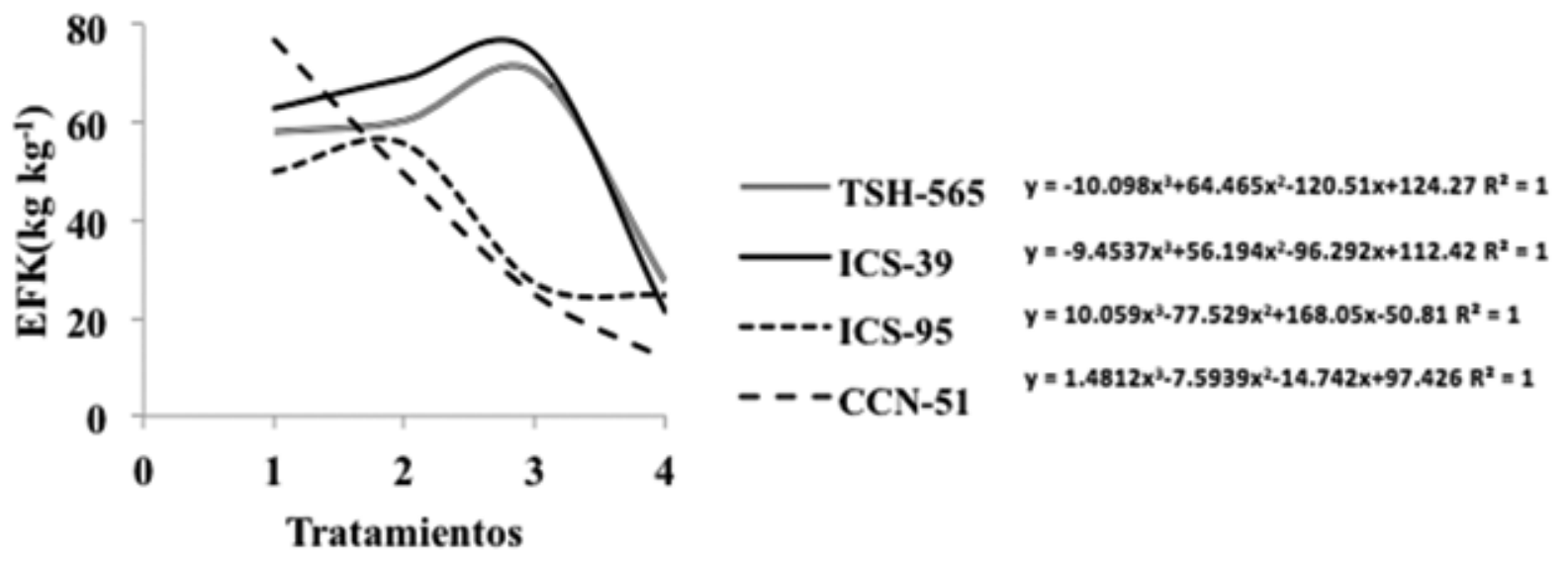

Figura 4. Eficiencia fisiológica de uso del potasio (EFK) para los clones TSH-565, ICS-39, ICS-95 y CCN-51. EFK: eficiencia fisiológica de uso de potasio

El clon CCN-51 mostro la mayor EFK, como también, la menor EFK (11,75 kg kg-1) en el T4, lo que sugiere que las altas dosis de potasio (T4) para los clones evaluados disminuyen la eficiencia fisiológica de uso del nutriente.

\section{Conclusiones}

La mayor eficiencia fisiológica de uso de nutrientes N-P-K se presentó en el tratamiento uno (T1) y la menor EF con la dosis nutricional más alta (T4), lo cual sugiere que a mayor dosis nutricional disminuye la capacidad de producción de grano seco de cacao por unidad de nutriente absorbido; así mismo, se evidencia que el clon CCN-51 muestra mayor habilidad de producción de grano seco por cada unidad de $\mathrm{N}$ y $\mathrm{P}$ absorbido, mientras que el clon ICS-39 presenta mayor producción de grano seco por cada unidad de K, sin embargo, no es generalizada una mayor habilidad de absorción en los clones autocompatibles con respecto a los clones autoincompatibles.

\section{Agradecimientos}

Los autores agradecen al Grupo de Investigación: "Uso y Manejo de Suelos y Aguas con énfasis en Degradación de Suelos" de la Universidad Nacional de Colombia, sede Palmira; y a la Federación Nacional de Cacaoteros de Colombia, Fedecacao. 


\section{Literatuta Citada}

1. Aguilar, C.C., Escalante, J.A.E. \& Aguilar, I. M. (2015). Análisis de crecimiento y rendimiento de maíz en clima cálido en función del genotipo, biofertilizante y nitrógeno. Terra Latinoam. 33:51-62.

2. Akinkunmi, E. A. (2006). Strategies for improving crops Use-Efficiencies of fertilizer nutrients in sustainable agricultural systems. Journal of nutrition 5(2): 185-193.

3. Allen, J. B. (1987). London Cocoa Trade Amazon Project. Final report phase 2. Special Issue of Cocoa Growers Bull. 39.

4. Baligar, V.C., Fageria, N.K. \& He, Z. L. (2001). Nutrient Use Efficiency in Plant, Communications in Soil Science and Plant Analysis, 32(7): 921 - 950.

5. Fageria, N. K., O. P. de Morais, \& A. B. dos Santos. (2010). Nitrogen use efficiency in upland rice genotypes. Journal of Plant Nutrition, 33:1696 - 1711.

6. Fan Jian-Bo., Zhang Ya-Li., D. Turner., Duan Yin-Hua, Wang Dong-Sheng \& Shen Qi-Rong. (2010). Root Physiological and Morphological Characteristics of Two Rice Cultivars with Different Nitrogen-Use Efficiency. Pedosphere 20(4): 446-455.

7. García, B. C. (1997). Cacao (Theobroma cacao L.). (433 p). Palmira, Colombia: Universidad Nacional de Colombia. Sede Palmira.

8. IBM (International Business Machines). (2011). Manual del usuario del sistema básico de IBM SPSS stadistic 20. $30 \mathrm{p}$.

9. ICA (Instituto Colombiano Agropecuario). (1992). Fertilización en diversos cultivos. Quinta Aproximación. Manual de Asistencia Técnica No.25. Centro de Investigación Tibaitata. Editorial Ediciones. 64 p.

10. Kumar, N. F. (2000). Eficiência do uso de potássio pelos genótipos de arroz de terras altas. Pesq. agropec. bras., Brasilia, 35 (10):2115-2120.

11. Lija, M., Osumanu A. H. \& Kasim, S. (2014). Maize (Zea mays L.) nutrient use efficiency as affected by formulated fertilizer with Clinoptilolite Zeolite. J. Food Agric. 2014. 26 (3): 284-292.
12. Mora, R. J., Burbano, O. H. \& Ballesteros, P. W. (2011). Efecto de la fertilización con diversas fuentes sobre el rendimiento de cacao. Rev. Cien. Agríc. 28(2):81 - 94.

13. Puentes, P.Y.J, Menjivar, J.C.F. \& Aranzazu, F. H. (2016). Concentración de nutrientes en hojas, una herramienta para el diagnóstico nutricional en cacao. Agron. Mesoam. 27(2):329-336.

14. Puentes, P.Y.J., F.J.C. Menjivar, \& Aránzazu, H.F. (2014a). Eficiencias en el uso de nitrógeno, fósforo y potasioen clones de cacao (Theobroma cacao L.). Bioagro 26:99-106.

15. Puentes, P.Y.J., J.C. Menjivar, A. Gómez \& Aranzazu, F. (2014b). Absorción y distribución de nutrientes en clones de cacao y sus efectos en el rendimiento. Acta Agron. 63:145-152.

16. Puerto, G.O., F.J.C. Menjivar, S. Mejía de T. \& Puentes, P.Y.J. (2014). Influencia del potasio en el cultivo de la vid (Vitis labrusca) cv. ISABELLA. Inf. Téc.78:148-154.

17. Rasche, J. W. A., Gómez V. A. L., Cabral, C. C. A., Muller, E. A. \& Schaefer, G. L. (2012). Potassium application in sugarcane varieties: effects on sugarcane yield and borer attack. Investig. Agrar., 14 (2):93-100.

18. Roberts, T. L. (2008). Improving Nutrient Use Efficiency. J.Agric 32:177-182.

19. Rouanet, M. J. L. (1994). Eficiencia fisiológica de nitrógeno por cultivos anuales en futura agricultura sustentable. Agricultura Técnica (Chile) 54 (2):169 - 179.

20. Soratto R. P., Pilon, C., Adalton M. F. \& Moreno, L.A. (2015). Phosphorus Uptake, Use Efficiency, and Response of Potato Cultivars to Phosphorus Levels. Potato Research 58:121-134.

21. Xu, X., Liu, X., He, P., Johnston, A.M., Zhao, S., Qiu S. et al. (2015) Yield Gap, Indigenous Nutrient Supply and Nutrient Use Efficiency for Maize in China. PLoS ONE 10(10): e0140767.
Conflicto de Intereses Los autores declaran no tener ningún conflicto de intereses 